\title{
Positive Periodic Solutions of Nonautonomous Delayed Predator-Prey System with Pulse Controls
}

\author{
Bin Wang, Shunqiang Shi, Ping Luo \\ Department of Mathematics, Xingyi Normal University for Nationalities Xingyi, China \\ Email: xywangbin@yeah.net
}

How to cite this paper: Wang, B., Shi, S.Q. and Luo, P. (2021) Positive Periodic Solutions of Nonautonomous Delayed Predator-Prey System with Pulse Controls. Journal of Applied Mathematics and Physics, 9, 1452-1467.

https://doi.org/10.4236/jamp.2021.97098

Received: June 5, 2021

Accepted: July 9, 2021

Published: July 12, 2021

Copyright ( 2021 by author(s) and Scientific Research Publishing Inc. This work is licensed under the Creative Commons Attribution International License (CC BY 4.0).

http://creativecommons.org/licenses/by/4.0/

\begin{abstract}
In this paper, a biological model for two predators and one prey with impulses and periodic delays is considered. By assuming that one predator consumes prey according to Holling II functional response while the other predators consume prey according to the Beddington-DeAngelis functional response, based on the coincidence degree theory, the existence of positive periodic solutions of nonautonomous predator-prey system with impulses and periodic delays is obtained under suitable conditions.
\end{abstract}

\section{Keywords}

Positive Periodic Solution, Predator-Prey, Impulses, Delays, Continuation Theorem

\section{(c) (i) Open Access}

\section{Introduction}

In the early 1920s, the work with respect to predator-prey systems [1] [2] [3] was done by Lotka [4] and Volterra [5], they concluded that the coexistence of two or more predators competing for fewer prey resources is impossible, which was later known as the principle of competitive exclusion. For example, in the case of two predators competing for a single prey species, one considers the following Lotka-Volterra predator-prey model

$$
\left\{\begin{array}{l}
\dot{x}(t)=r x\left[1-\frac{x}{k}\right]-a x y-A x z, \\
\dot{y}(t)=y[-d+e x], \\
\dot{z}(t)=z[-D+E x],
\end{array}\right.
$$


where $y(t)$ and $z(t)$ are the densities of the two predators and $x(t)$ is the density of the prey. By assuming that both predators consume prey according to Holling II functional response, then system (1.1) was modified to the following predator-prey system

$$
\left\{\begin{array}{l}
\dot{x}(t)=r x\left[1-\frac{x}{k}\right]-\frac{a x y}{1+b x}-\frac{A x z}{1+B x}, \\
\dot{y}(t)=y\left[-d+\frac{e x}{1+b x}\right], \\
\dot{z}(t)=z\left[-D+\frac{E x}{1+B x}\right] .
\end{array}\right.
$$

Based on dynamical system techniques and the geometrical singular perturbation theory, Liu, Xiao and Yi [6] showed the coexistence of predators and prey of system (1.2) which happened along a stable periodic orbit in the positive octant of $\mathbb{R}^{3}$.

By assuming that one predator consumes prey according to the Holling II functional response and the other predators consume prey according to the Beddington-DeAngelis functional response, then system (1.1) was modified to the following predator-prey system

$$
\left\{\begin{array}{l}
\dot{x}(t)=r x\left[1-\frac{x}{k}\right]-\frac{a x y}{1+b x}-\frac{A x z}{1+B x+C z}, \\
\dot{y}(t)=y\left[-d+\frac{e x}{1+b x}\right], \\
\dot{z}(t)=z\left[-D+\frac{E x}{1+B x+C z}\right] .
\end{array}\right.
$$

In [7], system (1.4) was studied with variable coefficient and periodic delays. The conditions of the existence of positive periodic solutions were given.

$$
\left\{\begin{aligned}
\dot{x}(t)= & r(t) x(t)\left[1-\frac{x(t)}{k}\right] \\
& -\frac{a(t) x(t) y(t)}{1+b x(t)}-\frac{A(t) x(t) z(t)}{1+B x(t)+C z(t)}, \\
\dot{y}(t)= & y(t)\left[-d(t)+\frac{e(t) x(t-\tau(t))}{1+b x(t-\tau(t))}\right], \\
\dot{z}(t)= & z(t)\left[-D(t)+\frac{E(t) x(t-\sigma(t))}{1+B x(t-\sigma(t))+C z(t-\sigma(t))}\right],
\end{aligned}\right.
$$

In the system based on the (1.4), taking into account the artificial interference, such as regularly input of predators and prey, this motivates us to consider the following system (1.5) with impulses. 


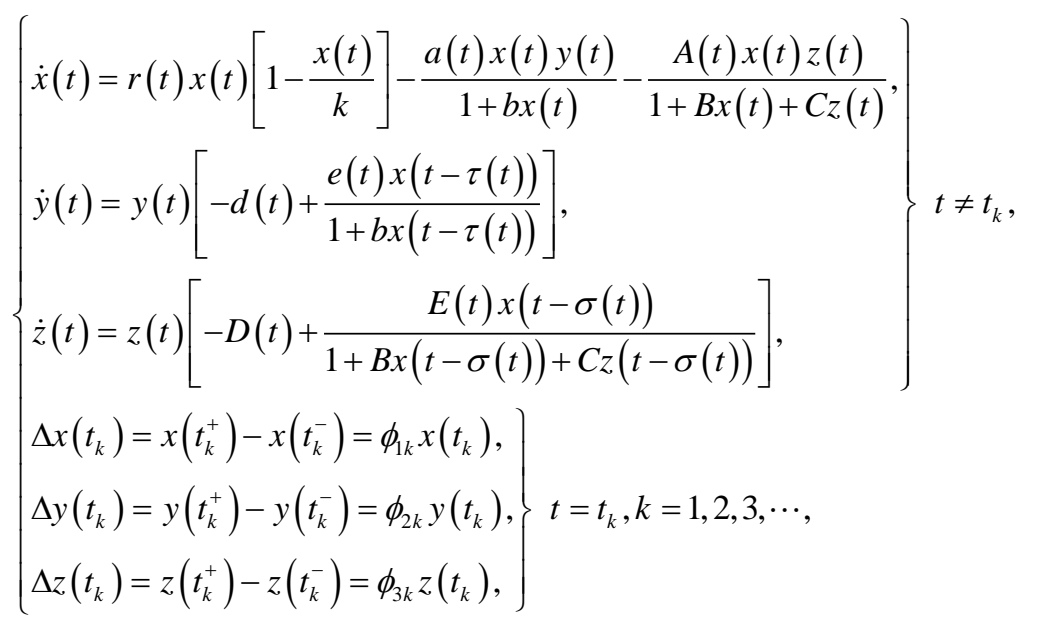

where $x(t)$ stands for the prey's density, $y(t)$ and $z(t)$ stand for the predators' densities respectively. One of the reasons for introducing a delay into a predator-prey system is that the rate of reproduction of predators depends on the rate at which they have consumed the prey in the past, and this idea is well justified [8] [9] [10]. Usually Hopf bifurcation theory is used to investigate the existence of periodic solutions for predator-prey system with a single constant delay [11] [12] [13]. Recently, the geometrical singular perturbation theory [14] has been employed by Lin and Yuan [15] [16] to study the existence of periodic solutions for predator-prey systems with a single delay or multiple delays. In this paper, we shall justify the existence of periodic solutions for predator-prey system (1.5) with impulses and periodic delays.

The main contribution of this paper is to obtain the existence of positive periodic solutions of nonautonomous delayed predator-prey system with pulse controls by using the method of the continuation theorem of Gaines and Mawhin. The rest of the paper is arranged as follows. In Section 2, we introduce some notation and concepts for the continuation theorem of coincidence degree, give some necessary definitions and lemmas. In Section 3, we establish new conditions for the existence of periodic solutions for the system (1.5). In Section 4, some lemmas are proved. Some conclusions are given in Section 5.

\section{Notations and Preliminaries}

Let $\mathbb{Z}, \mathbb{Z}^{+}$and $\mathbb{R}$ be the sets of all integers, positive integers and real numbers, respectively, and $\mathbb{R}^{+}=[0,+\infty)$.

Assume that

(H1) $r(t), a(t), A(t), d(t), e(t), D(t)$ and $E(t)$ are positive $\omega$-periodic continuous functions, $\tau(t)$ and $\sigma(t)$ are nonnegative $\omega$-periodic continuous functions, $k, b, B$ and $C$ are positive constants;

(H2) there exists an integer $p \geq 1$ such that $[0, \omega] \cap\left\{t_{k}\right\}=\left\{t_{1}, t_{2}, \cdots, t_{p}\right\}$, $0=t_{0}<t_{1}<t_{2}<\cdots<t_{k}<\cdots$, and $\lim _{k \rightarrow \infty} t_{k}=\infty, t_{k+p}=t_{k}+\omega$, $\phi_{i(k+p)}=\phi_{i k}(i=1,2,3), \quad k=1,2,3, \cdots$. Furthermore, a natural constraint is $\phi_{i k}>-1,(i=1,2,3), \quad k=1,2,3, \cdots$; 


$$
\begin{aligned}
& \text { (H3) } x\left(t_{k}^{-}\right)=\lim _{t \rightarrow t_{k}-0} x(t)=x\left(t_{k}\right), y\left(t_{k}^{-}\right)=\lim _{t \rightarrow t_{k}-0} y(t)=y\left(t_{k}\right), \\
& z\left(t_{k}^{-}\right)=\lim _{t \rightarrow t_{k}-0} z(t)=z\left(t_{k}\right) .
\end{aligned}
$$

Let

$$
P C_{\omega}=\left\{\psi: \mathbb{R}^{+} \rightarrow \mathbb{R} \mid \begin{array}{l}
(\mathrm{i}) \psi(t) \text { is continuous for } t \neq t_{k} \text { and } \omega \text {-periodic; } \\
(\mathrm{ii}) \lim _{s \rightarrow t_{k}-0} \psi(s)=\psi\left(t_{k}\right) \text { and } \lim _{s \rightarrow t_{k}+0} \psi(s)=\psi\left(t_{k}^{+}\right) \text {exist }
\end{array}\right\} .
$$

Moreover, we denote $P C_{\omega}^{1}=\left\{\psi \in P C_{\omega}: \psi^{\prime} \in P C_{\omega}\right\}$.

Before stating our main result, for the sake of convenience and simplicity, we denote

$$
\begin{gathered}
\bar{f}=\frac{1}{\omega} \int_{0}^{\omega} f(t) \mathrm{d} t \quad \text { for any } f(t) \in P C_{\omega} . \\
\phi_{i}=\sum_{k=1}^{p} \ln \left(1+\phi_{i k}\right), \quad \Phi_{i}=\phi_{i}+\left|\phi_{i}\right|, \quad i=1,2,3 .
\end{gathered}
$$

Definition 2.1. ([17]). The set $\mathcal{F}$ is said to be quasi equi-continuous in $[0, \omega]$ if for any $\varepsilon>0$ there exists $\delta>0$ such that if $x \in \mathcal{F}, k \in \mathbb{Z}^{+}$, $t^{\prime}, t^{\prime \prime} \in\left(t_{k-1}, t_{k}\right] \cap[0, \omega]$, and $\left|t^{\prime}-t^{\prime \prime}\right|<\delta$, then

$$
\left|x\left(t^{\prime}\right)-x\left(t^{\prime \prime}\right)\right|<\varepsilon
$$

Lemma 1. (Compactness criterion, Lemma 2.4 of [17]). The set $\mathcal{F} \subset P C_{\omega}$ is relatively compact if and only if:

a) $\mathcal{F}$ is bounded, that is, there exists some $M>0$ such that $\|x\| \leq M$ for each $x \in \mathcal{F}$;

b) $\mathcal{F}$ is quasi equi-continuous in $[0, \omega]$.

For convenience, we summarize a few concepts from the book by Gaines and Mawhin [18].

Let $X$ and $Z$ be real Banach spaces. Let $L: \operatorname{Dom} L \subset X \rightarrow Z$ be a linear mapping and $N: X \rightarrow Z$ be a continuous mapping. The mapping $L$ will be called a Fredholm mapping of index zero if $\operatorname{dimKer} L=\operatorname{codim} \operatorname{Im} L<+\infty$ and $\operatorname{Im} L$ is closed in $Z$. If $L$ is a Fredholm mapping of index zero, then there exist continuous projectors $P: X \rightarrow X$ and $Q: Z \rightarrow Z$ such that $\operatorname{Im} P=\operatorname{Ker} L$ and $\operatorname{Im} L=\operatorname{Ker} Q=\operatorname{Im}(I-Q)$. It follows that $\left.L\right|_{\text {DomL } L \text { KerP }}:(I-P) X \rightarrow \operatorname{Im} L$ is invertible and its inverse is denoted by $K_{P}$. If $\Omega$ is a bounded open subset of $X$, the mapping $N$ is called $L$-compact on $\bar{\Omega}$ if $(Q N)(\bar{\Omega})$ is bounded and $K_{P}(I-Q) N: \bar{\Omega} \rightarrow X$ is compact. Because $\operatorname{Im} Q$ is isomorphic to $\operatorname{Ker} L$, there exists an isomorphism $J: \operatorname{Im} Q \rightarrow \operatorname{Ker} L$.

In the proof of our existence result, we need the following continuation theorem.

Lemma 2. (Continuation Theorem, Gaines and Mawhin [18]). Let $L$ be a Fredholm mapping of index zero and let $N$ be $L$-compact on $\bar{\Omega}$. Suppose
a) for each $\lambda \in(0,1)$, every solution $x$ of $L x=\lambda N x$ is such that $x \notin \partial \Omega$;
b) $Q N x \neq 0$ for each $x \in \operatorname{Ker} L \cap \partial \Omega$;
c) $\operatorname{deg}\{J Q N, \Omega \bigcap \operatorname{Ker} L, 0\} \neq 0$. 
Then the equation $L x=N x$ has at least one solution lying in $\operatorname{Dom} L \cap \bar{\Omega}$.

In order to transform (1.5) to the equivalent operator equation, we carry out the change of variable $x(t)=\mathrm{e}^{u_{1}(t)}, y(t)=\mathrm{e}^{u_{2}(t)}, z(t)=\mathrm{e}^{u_{3}(t)}$, then (1.5) has the form

$$
\left\{\begin{array}{l}
\dot{u}_{1}(t)=r(t)\left[1-\frac{\mathrm{e}^{u_{1}(t)}}{k}\right]-\frac{a(t) \mathrm{e}^{u_{2}(t)}}{1+b \mathrm{e}^{u_{1}(t)}}-\frac{A(t) \mathrm{e}^{u_{3}(t)}}{1+B \mathrm{e}^{u_{1}(t)}+C \mathrm{e}^{u_{3}(t)}} \triangleq F_{1}(t), \\
\dot{u}_{2}(t)=-d(t)+\frac{e(t) \mathrm{e}^{u_{1}(t-\tau(t))}}{1+b \mathrm{e}^{u_{1}(t-\tau(t))}} \triangleq F_{2}(t), \\
\dot{u}_{3}(t)=\left[-D(t)+\frac{E(t) \mathrm{e}^{u_{1}(t-\sigma(t))}}{1+B \mathrm{e}^{u_{1}(t-\sigma(t))}+C \mathrm{e}^{u_{3}(t-\sigma(t))}}\right] \triangleq F_{3}(t), \\
\Delta u_{i}\left(t_{k}\right)=u_{i}\left(t_{k}^{+}\right)-u_{i}\left(t_{k}^{-}\right)=\ln \left(1+\phi_{i k}\right), t=t_{k},
\end{array}\right\} t \neq t_{k},
$$

where $i=1,2,3, k \in \mathbb{Z}^{+}$.

It is easy to see that if system (2.1) has one $\omega$-periodic solution $\left(u_{1}^{*}(t), u_{2}^{*}(t), u_{3}^{*}(t)\right)^{\mathrm{T}}$, then $\left(x^{*}(t), y^{*}(t), z^{*}(t)\right)^{\mathrm{T}}=\left(\mathrm{e}^{u_{1}^{*}(t)}, \mathrm{e}^{u_{2}^{*}(t)}, \mathrm{e}^{u_{3}^{*}(t)}\right)^{\mathrm{T}}$ is a positive $\omega$-periodic solution of system (1.5). Therefore, it suffices to prove system (2.1) has an $\omega$-periodic solution.

Let

$$
X=\left\{u=\left(u_{1}, u_{2}, u_{3}\right)^{\mathrm{T}} \mid u_{i} \in P C_{\omega}, i=1,2,3\right\}, \quad Z=X \times \mathbb{R}^{3 p}
$$

with the norm

$$
\|u\|_{X}=\sum_{i=1}^{3} \sup _{t \in[0, \omega]}\left|u_{i}(t)\right|, \quad \forall u=\left(u_{1}, u_{2}, u_{3}\right)^{\mathrm{T}} \in X
$$

and

$$
\|z\|_{Z}=\|u\|_{X}+\|y\|, \quad \forall z=(u, y) \in Z,
$$

where $\|\cdot\|$ is the Euclidean norm of $\mathbb{R}^{3 p}$. Then $X$ and $Z$ are Banach spaces when they are endowed with above norms.

Set $L: \operatorname{Dom} L \subset X \rightarrow Z, \quad L u=\left(\dot{u}, \Delta u\left(t_{1}\right), \cdots, \Delta u\left(t_{p}\right)\right)$ with

$\operatorname{Dom} L=\left\{u=\left(u_{1}, u_{2}, u_{3}\right)^{\mathrm{T}} \mid u_{i} \in P C_{\omega}, i=1,2,3\right\}, \quad Z=X \times \mathbb{R}^{3 p} \quad$ and $\quad N: X \rightarrow Z$ as

$$
\begin{gathered}
N\left(\begin{array}{l}
u_{1} \\
u_{2} \\
u_{3}
\end{array}\right)=\left(\begin{array}{c}
F_{1}(t), \ln \left(1+\phi_{11}\right), \cdots, \ln \left(1+\phi_{1 p}\right) \\
F_{2}(t), \ln \left(1+\phi_{21}\right), \cdots, \ln \left(1+\phi_{2 p}\right) \\
F_{3}(t), \ln \left(1+\phi_{31}\right), \cdots, \ln \left(1+\phi_{3 p}\right)
\end{array}\right), \\
\forall\left(u_{1}, u_{2}, u_{3}\right)^{\mathrm{T}} \in X .
\end{gathered}
$$

Using this notation we may rewrite (2.1) in the equivalent form $L u=N u, u \in X$. Clearly, $\operatorname{Ker} L=\mathbb{R}^{3}, \quad \operatorname{Im} L=\left\{z=\left(F, \gamma_{1}, \cdots, \gamma_{p}\right) \in Z: \int_{0}^{\omega} F(t) \mathrm{d} t+\sum_{k=1}^{p} \gamma_{k}=0\right\} \quad$ is closed in $Z$, and $\operatorname{dimKer} L=\operatorname{codim} \operatorname{Im} L=3$. Thus, $L$ is a Fredholm mapping of 
index zero. Define two projections $P: X \rightarrow X$ and $Q: Z \rightarrow Z$ as

$$
\begin{gathered}
P u=\bar{u}, \quad u \in X, \\
Q z=Q\left(u, \gamma_{1}, \cdots, \gamma_{p}\right)=\left(\bar{u}+\frac{1}{\omega} \sum_{k=1}^{p} \gamma_{k}, 0, \cdots, 0\right) .
\end{gathered}
$$

It is trivial to show that $P, Q$ are continuous projections such that

$$
\operatorname{Im} P=\operatorname{Ker} L, \operatorname{Ker} Q=\operatorname{Im} L=\operatorname{Im}(I-Q),
$$

and hence, the generalized inverse $K_{P}$ exists. In order to derive the expression of $K_{P}: \operatorname{Im} L \rightarrow \operatorname{Ker} P \cap \operatorname{Dom} L$, we take $z=\left(F, \gamma_{1}, \cdots, \gamma_{p}\right) \in \operatorname{Im} L$, then there exists $u \in \operatorname{Dom} L \subset X$ such that

$$
\left\{\begin{array}{l}
u(t)=F(t), t \neq t_{k} \\
\Delta u(t)=\gamma_{k}, t=t_{k}, k \in \mathbb{Z}^{+}
\end{array}\right.
$$

A direct integration gives

$$
u(t)=u(0)+\int_{0}^{t} F(s) \mathrm{d} s+\sum_{t_{k}<t} \gamma_{k}
$$

Note that $u \in \operatorname{Ker} P$, namely, $\int_{0}^{\omega} u(s) \mathrm{d} s=0$, together with (2.2) implies

$$
\omega u(0)+\int_{0}^{\omega} \int_{0}^{t} F(s) \mathrm{d} s \mathrm{~d} t+\int_{0}^{\omega} \sum_{t_{k}<t} \gamma_{k} \mathrm{~d} t=0
$$

then

$$
u(t)=\int_{0}^{t} F(s) \mathrm{d} s+\sum_{t_{k}<t} \gamma_{k}-\frac{1}{\omega} \int_{0}^{\omega} \int_{0}^{t} F(s) \mathrm{d} s \mathrm{~d} t-\sum_{k=1}^{p} \gamma_{k}+\frac{1}{\omega} \sum_{k=1}^{p} \gamma_{k} t_{k} .
$$

Therefore, for $u \in X$, one has

$$
Q N u=\frac{1}{\omega}\left(\begin{array}{l}
\int_{0}^{\omega} F_{1}(t) \mathrm{d} t+\sum_{k=1}^{p} \ln \left(1+\phi_{1 k}\right), 0, \cdots, 0 \\
\int_{0}^{\omega} F_{2}(t) \mathrm{d} t+\sum_{k=1}^{p} \ln \left(1+\phi_{2 k}\right), 0, \cdots, 0 \\
\int_{0}^{\omega} F_{3}(t) \mathrm{d} t+\sum_{k=1}^{p} \ln \left(1+\phi_{3 k}\right), 0, \cdots, 0
\end{array}\right)
$$

and $K_{p}(I-Q) N: X \rightarrow X$,

$$
\begin{aligned}
K_{p}(I-Q) N u= & \left(\begin{array}{l}
\int_{0}^{t} F_{1}(t) \mathrm{d} t+\sum_{k=1}^{p} \ln \left(1+\phi_{1 k}\right) \\
\int_{0}^{t} F_{2}(t) \mathrm{d} t+\sum_{k=1}^{p} \ln \left(1+\phi_{2 k}\right) \\
\int_{0}^{t} F_{3}(t) \mathrm{d} t+\sum_{k=1}^{p} \ln \left(1+\phi_{3 k}\right)
\end{array}\right) \\
& +\left(\frac{1}{2}-\frac{t}{\omega}\right)\left(\begin{array}{l}
\int_{0}^{\omega} F_{1}(t) \mathrm{d} t+\sum_{k=1}^{p} \ln \left(1+\phi_{1 k}\right) \\
\int_{0}^{\omega} F_{2}(t) \mathrm{d} t+\sum_{k=1}^{p} \ln \left(1+\phi_{2 k}\right) \\
\int_{0}^{\omega} F_{3}(t) \mathrm{d} t+\sum_{k=1}^{p} \ln \left(1+\phi_{3 k}\right)
\end{array}\right)
\end{aligned}
$$




$$
-\frac{1}{\omega}\left(\begin{array}{l}
\int_{0}^{\omega} \int_{0}^{t} F_{1}(s) \mathrm{d} s \mathrm{~d} t+\omega \sum_{k=1}^{p} \ln \left(1+\phi_{1 k}\right)-\sum_{k=1}^{p} \ln \left(1+\phi_{1 k}\right) t_{k} \\
\int_{0}^{\omega} \int_{0}^{t} F_{2}(s) \mathrm{d} s \mathrm{~d} t+\omega \sum_{k=1}^{p} \ln \left(1+\phi_{2 k}\right)-\sum_{k=1}^{p} \ln \left(1+\phi_{2 k}\right) t_{k} \\
\int_{0}^{\omega} \int_{0}^{t} F_{3}(s) \mathrm{d} s \mathrm{~d} t+\omega \sum_{k=1}^{p} \ln \left(1+\phi_{3 k}\right)-\sum_{k=1}^{p} \ln \left(1+\phi_{3 k}\right) t_{k}
\end{array}\right)
$$

Clearly, $Q N$ and $K_{p}(I-Q) N$ are continuous. Using the Arzela-Ascoli theorem and Lemma 1 , it is not difficult to prove that $\overline{K_{p}(I-Q) N(\bar{\Omega})}$ is compact for any open bounded set $\Omega \subset X$. In addition, $Q N(\bar{\Omega})$ is bounded. Therefore, $N$ is $L$-compact on $\bar{\Omega}$ with any open bounded set $\Omega \subset X$.

Now we reach the position to search for an appropriate open bounded subset $\Omega \subset X$ for the application of the continuation theorem (Lemma 2). Corresponding to the operator equation $L x=\lambda N x, \lambda \in(0,1)$, we have

$$
\left\{\begin{array}{l}
\dot{u}_{1}(t)=\lambda\left[r(t)\left(1-\frac{\mathrm{e}^{u_{1}(t)}}{k}\right)-\frac{a(t) \mathrm{e}^{u_{2}(t)}}{1+b \mathrm{e}^{u_{1}(t)}}-\frac{A(t) \mathrm{e}^{u_{3}(t)}}{1+B \mathrm{e}^{u_{1}(t)}+C \mathrm{e}^{u_{3}(t)}}\right] \\
\dot{u}_{2}(t)=\lambda\left[-d(t)+\frac{e(t) \mathrm{e}^{u_{1}(t-\tau(t))}}{1+b \mathrm{e}^{u_{1}(t-\tau(t))}}\right], \\
\dot{u}_{3}(t)=\lambda\left[-D(t)+\frac{E(t) \mathrm{e}^{u_{1}(t-\sigma(t))}}{1+B \mathrm{e}^{u_{1}(t-\sigma(t))}+C \mathrm{e}^{u_{3}(t-\sigma(t))}}\right], \\
\Delta u_{i}\left(t_{k}\right)=u_{i}\left(t_{k}^{+}\right)-u_{i}\left(t_{k}^{-}\right)=\lambda \ln \left(1+\phi_{i k}\right), t=t_{k},
\end{array}\right\} t \neq t_{k},
$$

where $i=1,2,3, k \in \mathbb{Z}^{+}$.

Suppose that $u=\left(u_{1}, u_{2}, u_{3}\right)^{\mathrm{T}} \in X$ is an $\omega$-periodic solution of (2.3) for some $\lambda \in(0,1)$, integrating $(2.3)$ over $[0, \omega]$, we obtain

$$
\begin{gathered}
\int_{0}^{\omega}\left[r(t)\left(1-\frac{\mathrm{e}^{u_{1}(t)}}{k}\right)+\frac{a(t) \mathrm{e}^{u_{2}(t)}}{1+b \mathrm{e}^{u_{1}(t)}}+\frac{A(t) \mathrm{e}^{u_{3}(t)}}{1+B \mathrm{e}^{u_{1}(t)}+C \mathrm{e}^{u_{3}(t)}}\right] \mathrm{d} t=-\phi_{1}, \\
\int_{0}^{\omega}\left[-d(t)+\frac{e(t) \mathrm{e}^{u_{1}(t-\tau(t))}}{1+b \mathrm{e}^{u_{1}(t-\tau(t))}}\right]=-\phi_{2}, \\
\int_{0}^{\omega}\left[-D(t)+\frac{E(t) \mathrm{e}^{u_{1}(t-\sigma(t))}}{1+B \mathrm{e}^{u_{1}(t-\sigma(t))}+C \mathrm{e}^{u_{3}(t-\sigma(t))}}\right] \mathrm{d} t=-\phi_{3} .
\end{gathered}
$$

Therefore,

$$
\begin{gathered}
\int_{0}^{\omega}\left[\frac{r(t) \mathrm{e}^{u_{1}(t)}}{k}+\frac{a(t) \mathrm{e}^{u_{2}(t)}}{1+b \mathrm{e}^{u_{1}(t)}}+\frac{A(t) \mathrm{e}^{u_{3}(t)}}{1+B \mathrm{e}^{u_{1}(t)}+C \mathrm{e}^{u_{3}(t)}}\right] \mathrm{d} t=\bar{r} \omega+\phi_{1}, \\
\int_{0}^{\omega} \frac{\mathrm{e}(t) \mathrm{e}^{u_{1}(t-\tau(t))}}{1+b \mathrm{e}^{u_{1}(t-\tau(t))}} \mathrm{d} t=\bar{d} \omega-\phi_{2}, \\
\int_{0}^{\omega} \frac{E(t) \mathrm{e}^{u_{1}(t-\sigma(t))}}{1+B \mathrm{e}^{u_{1}(t-\sigma(t))}+C \mathrm{e}^{u_{3}(t-\sigma(t))}} \mathrm{d} t=\bar{D} \omega-\phi_{3} .
\end{gathered}
$$


It follows from (2.3)-(2.6) that

$$
\begin{aligned}
& \int_{0}^{\omega}\left|\dot{u}_{1}(t)\right| \mathrm{d} t \leq \int_{0}^{\omega} r_{1}(t) \mathrm{d} t+\int_{0}^{\omega}\left[\frac{r(t) \mathrm{e}^{u_{1}(t)}}{k}+\frac{a(t) \mathrm{e}^{u_{2}(t)}}{1+b \mathrm{e}^{u_{1}(t)}}+\frac{A(t) x(t) \mathrm{e}^{u_{3}(t)}}{1+B \mathrm{e}^{u_{1}(t)}+C \mathrm{e}^{u_{3}(t)}}\right] \mathrm{d} t \\
&= 2 \bar{r} \omega+\phi_{1}, \\
& \int_{0}^{\omega}\left|\dot{u}_{2}(t)\right| \mathrm{d} t \leq \int_{0}^{\omega} d(t) \mathrm{d} t+\int_{0}^{\omega} \frac{e(t) \mathrm{e}^{u_{1}(t-\tau(t))}}{1+b \mathrm{e}^{u_{1}(t-\tau(t))}} \mathrm{d} t=2 \bar{d} \omega-\phi_{2}, \\
& \int_{0}^{\omega}\left|\dot{u}_{3}(t)\right| \mathrm{d} t \leq \int_{0}^{\omega} D(t) \mathrm{d} t+\int_{0}^{\omega} \frac{E(t) \mathrm{e}^{u_{1}(t-\sigma(t))}}{1+B \mathrm{e}^{u_{1}(t-\sigma(t))}+C \mathrm{e}^{u_{3}(t-\sigma(t))}} \mathrm{d} t=2 \bar{D} \omega-\phi_{3} .
\end{aligned}
$$

Note that $\left(u_{1}(t), u_{2}(t), u_{3}(t)\right)^{\mathrm{T}} \in X$, then there exists $\xi_{i}, \eta_{i} \in[0, \omega]$, $i=1,2,3$ such that

$$
u_{i}\left(\xi_{i}\right)=\min _{t \in[0, \omega]} u_{i}(t), \quad u_{i}\left(\eta_{i}\right)=\max _{t \in[0, \omega]} u_{i}(t), \quad i=1,2,3 .
$$

The following three lemmas give priori estimates for the three components of the solution $\left(u_{1}(t), u_{2}(t), u_{3}(t)\right)^{\mathrm{T}} \in X \quad$ in system (2.3) and their proofs will be given in the next section.

Lemma 3. $\max _{t \in[0, \omega]}\left|u_{1}(t)\right| \leq \max \left\{\left|W_{11}\right|,\left|W_{12}\right|\right\} \triangleq W_{1}$, where

$$
\begin{aligned}
& W_{11}=\ln \left[\frac{\bar{d} \omega-\phi_{2}}{\bar{e} \omega-b\left(\bar{d} \omega-\phi_{2}\right)}\right]-2 \bar{r} \omega-\Phi_{1}, \\
& W_{12}=\ln \left[\frac{\bar{d} \omega-\phi_{2}}{\bar{e} \omega-b\left(\bar{d} \omega-\phi_{2}\right)}\right]+2 \bar{r} \omega+\Phi_{1} .
\end{aligned}
$$

Lemma 4. $\max _{t \in[0, \omega]}\left|u_{3}(t)\right| \leq \max \left\{\left|W_{31}\right|,\left|W_{32}\right|\right\} \triangleq W_{3}$, where

$$
\begin{aligned}
& W_{31}=\ln \frac{\left[\bar{E} \omega-B\left(\bar{D} \omega-\phi_{3}\right)\right] \mathrm{e}^{W_{11}}-\bar{D} \omega+\phi_{3}}{C\left(\bar{D} \omega-\phi_{3}\right)}-2 \bar{D} \omega+\phi_{3}-\left|\phi_{3}\right|, \\
& W_{32}=\ln \frac{\left[\bar{E} \omega-B\left(\bar{D} \omega-\phi_{3}\right)\right] \mathrm{e}^{W_{12}}-\bar{D} \omega+\phi_{3}}{C\left(\bar{D} \omega-\phi_{3}\right)}-2 \bar{D} \omega+\phi_{3}-\left|\phi_{3}\right| .
\end{aligned}
$$

Lemma 5. $\max _{t \in[0, \omega]}\left|u_{2}(t)\right| \leq \max \left\{\left|W_{21}\right|,\left|W_{22}\right|\right\} \triangleq W_{2}$, where

$$
\begin{aligned}
W_{21}= & \ln \left\{\frac{\bar{e} \omega}{\bar{a} \omega\left(\bar{d} \omega-\phi_{2}\right)}\left[\mathrm{e}^{W_{11}}\left(\left(\bar{r} \omega+\phi_{1}\right)-\frac{\bar{r} \omega \mathrm{e}^{W_{12}}}{k}\right)-\frac{\bar{A} \omega\left(\bar{D} \omega-\phi_{3}\right) \mathrm{e}^{W_{32}}}{\bar{E} \omega}\right]\right\} \\
& -2 \bar{d} \omega+\phi_{2}-\left|\phi_{2}\right|, \\
W_{22}= & \ln \left\{\frac{\bar{e} \omega}{\bar{a} \omega\left(\bar{d} \omega-\phi_{2}\right)}\left[\mathrm{e}^{W_{12}}\left(\left(\bar{r} \omega+\phi_{1}\right)-\frac{\bar{r} \omega \mathrm{e}^{W_{11}}}{k}\right)-\frac{\bar{A} \omega\left(\bar{D} \omega-\phi_{3}\right) \mathrm{e}^{W_{31}}}{\bar{E} \omega}\right]\right\} \\
& +2 \bar{d} \omega-\phi_{2}+\left|\phi_{2}\right| .
\end{aligned}
$$

\section{Proof of Lemmas}

In this section, we give the proof of Lemmas 4-6. 
Assume that

(H4) $\bar{d} \omega-\phi_{2}>0, \bar{e} \omega-b\left(\bar{d} \omega-\phi_{2}\right)>0, \bar{r} \omega+\phi_{1}>0$, $\left[\bar{E} \omega-B\left(\bar{D} \omega-\phi_{3}\right)\right] \mathrm{e}^{W_{11}}>\bar{D} \omega-\phi_{3}>0$, (H5) $\left[\mathrm{e}^{W_{11}}\left(\left(\bar{r} \omega+\phi_{1}\right)-\frac{\bar{r} \omega \mathrm{e}^{W_{12}}}{k}\right)-\frac{\bar{A} \omega\left(\bar{D} \omega-\phi_{3}\right) \mathrm{e}^{W_{32}}}{\bar{E} \omega}\right]>0$,

(H6) $\left[\mathrm{e}^{W_{12}}\left(\left(\bar{r} \omega+\phi_{1}\right)-\frac{\bar{r} \omega \mathrm{e}^{W_{11}}}{k}\right)-\frac{\bar{A} \omega\left(\bar{D} \omega-\phi_{3}\right) \mathrm{e}^{W_{31}}}{\bar{E} \omega}\right]>0$, where

$$
\begin{gathered}
W_{11}=\ln \left[\frac{\bar{d} \omega-\phi_{2}}{\bar{e} \omega-b\left(\bar{d} \omega-\phi_{2}\right)}\right]-2 \bar{r} \omega-\Phi_{1}, \\
W_{12}=\ln \left[\frac{\bar{d} \omega-\phi_{2}}{\bar{e} \omega-b\left(\bar{d} \omega-\phi_{2}\right)}\right]+2 \bar{r} \omega+\Phi_{1}, \\
W_{31}=\ln \frac{\left[\bar{E} \omega-B\left(\bar{D} \omega-\phi_{3}\right)\right] \mathrm{e}^{W_{11}}-\bar{D} \omega+\phi_{3}}{C\left(\bar{D} \omega-\phi_{3}\right)}-2 \bar{D} \omega+\phi_{3}-\left|\phi_{3}\right|, \\
W_{32}=\ln \frac{\left[\bar{E} \omega-B\left(\bar{D} \omega-\phi_{3}\right)\right] \mathrm{e}^{W_{12}}-\bar{D} \omega+\phi_{3}}{C\left(\bar{D} \omega-\phi_{3}\right)}-2 \bar{D} \omega+\phi_{3}-\left|\phi_{3}\right| .
\end{gathered}
$$

Proof of Lemma 4. By (2.5) and (2.10), we have

$$
\frac{\bar{e} \omega \mathrm{e}^{u_{1}\left(\xi_{1}\right)}}{1+b \mathrm{e}^{u_{1}\left(\xi_{1}\right)}} \leq \bar{d} \omega-\phi_{2},
$$

that is

$$
\frac{\mathrm{e}^{u_{1}\left(\xi_{1}\right)}}{1+b \mathrm{e}^{u_{1}\left(\xi_{1}\right)}} \leq \frac{\bar{d} \omega-\phi_{2}}{\bar{e} \omega}
$$

It follows that

$$
u_{1}\left(\xi_{1}\right) \leq \ln \left[\frac{\bar{d} \omega-\phi_{2}}{\bar{e} \omega-b\left(\bar{d} \omega-\phi_{2}\right)}\right] \triangleq G_{12}
$$

By (3.2), we have

$$
u_{1}(t) \leq u_{1}\left(\xi_{1}\right)+\int_{0}^{\omega}\left|\dot{u}_{1}(t)\right| \mathrm{d} t+\left|\phi_{1}\right| \leq G_{12}+2 \omega \bar{r}+\Phi_{1} \triangleq W_{12} .
$$

On the other hand, we have

$$
\bar{d} \omega-\phi_{2} \leq \frac{\bar{e} \omega \mathrm{e}^{u_{1}\left(\eta_{1}\right)}}{1+b \mathrm{e}^{u_{1}\left(\eta_{1}\right)}},
$$

that is

$$
\frac{\bar{d} \omega-\phi_{2}}{\bar{e} \omega} \leq \frac{\mathrm{e}^{u_{1}\left(\eta_{1}\right)}}{1+b \mathrm{e}^{u_{1}\left(\eta_{1}\right)}} .
$$

It follows that 


$$
u_{1}\left(\eta_{1}\right) \geq \ln \left[\frac{\bar{d} \omega-\phi_{2}}{\bar{e} \omega-b\left(\bar{d} \omega-\phi_{2}\right)}\right] \triangleq G_{11} .
$$

By (2.3) and (3.5), we have

$$
u_{1}(t) \geq u_{1}\left(\eta_{1}\right)-\int_{0}^{\omega}\left|\dot{u}_{1}(t)\right| \mathrm{d} t-\left|\phi_{1}\right| \geq G_{11}-2 \bar{r} \omega-\Phi_{1} \triangleq W_{11} .
$$

Therefore, by (3.3) and (3.6), we have

$$
\max _{t \in[0, \omega]}\left|u_{1}(t)\right| \leq \max \left\{\left|W_{11}\right|,\left|W_{12}\right|\right\} .
$$

This completes the proof of Lemma 4.

Proof of Lemma 5. By (2.6) and (2.10), we have

$$
\bar{D} \omega-\phi_{3} \geq \int_{0}^{\omega} \frac{E(t) \mathrm{e}^{u_{1}(t-\sigma(t))}}{1+B \mathrm{e}^{u_{1}(t-\sigma(t))}+C \mathrm{e}^{u_{3}\left(\eta_{3}\right)}} \mathrm{d} t \geq \frac{\bar{E} \omega \mathrm{e}^{u_{1}\left(\xi_{1}\right)}}{1+B \mathrm{e}^{u_{1}\left(\xi_{1}\right)}+C \mathrm{e}^{u_{3}\left(\eta_{3}\right)}},
$$

that is

$$
\frac{\mathrm{e}^{u_{1}\left(\xi_{1}\right)}}{1+B \mathrm{e}^{u_{1}\left(\xi_{1}\right)}+C \mathrm{e}^{u_{3}\left(\eta_{3}\right)}} \leq \frac{\bar{D} \omega-\phi_{3}}{\bar{E} \omega} .
$$

It follows that

$$
\mathrm{e}^{u_{3}\left(\eta_{3}\right)} \geq \frac{\left[\bar{E} \omega-B\left(\bar{D} \omega-\phi_{3}\right)\right] \mathrm{e}^{u_{1}\left(\xi_{1}\right)}-\bar{D} \omega+\phi_{3}}{C\left(\bar{D} \omega-\phi_{3}\right)} .
$$

By (3.6), we have

$$
\mathrm{e}^{u_{3}\left(\eta_{3}\right)} \geq \frac{\left[\bar{E} \omega-B\left(\bar{D} \omega-\phi_{3}\right)\right] \mathrm{e}^{W_{11}}-\bar{D} \omega+\phi_{3}}{C\left(\bar{D} \omega-\phi_{3}\right)} .
$$

We have

$$
u_{3}\left(\eta_{3}\right) \geq \ln \frac{\left[\bar{E} \omega-B\left(\bar{D} \omega-\phi_{3}\right)\right] \mathrm{e}^{W_{11}}-\bar{D} \omega+\phi_{3}}{C\left(\bar{D} \omega-\phi_{3}\right)} \triangleq G_{31} .
$$

By (3.8), we have

$$
u_{3}(t) \geq u_{3}\left(\eta_{3}\right)-\int_{0}^{\omega}\left|\dot{u}_{3}(t)\right| \mathrm{d} t-\left|\phi_{3}\right| \geq G_{31}-2 \bar{D} \omega+\phi_{3}-\left|\phi_{3}\right| \triangleq W_{31} .
$$

On the other hand, we have

$$
\bar{D} \omega-\phi_{3} \leq \int_{0}^{\omega} \frac{E(t) \mathrm{e}^{u_{1}(t-\sigma(t))}}{1+B \mathrm{e}^{u_{1}(t-\sigma(t))}+C \mathrm{e}^{u_{3}\left(\xi_{3}\right)}} \mathrm{d} t \leq \frac{\bar{E} \omega \mathrm{e}^{u_{1}\left(\eta_{1}\right)}}{1+B \mathrm{e}^{u_{1}\left(\eta_{1}\right)}+C \mathrm{e}^{u_{3}\left(\xi_{3}\right)}},
$$

that is

$$
\frac{\mathrm{e}^{u_{1}\left(\eta_{1}\right)}}{1+B \mathrm{e}^{u_{1}\left(\eta_{1}\right)}+C \mathrm{e}^{u_{3}\left(\xi_{3}\right)}} \geq \frac{\bar{D} \omega-\phi_{3}}{\bar{E} \omega} .
$$

It follows that

$$
\mathrm{e}^{u_{3}\left(\xi_{3}\right)} \leq \frac{\left[\bar{E} \omega-B\left(\bar{D} \omega-\phi_{3}\right)\right] \mathrm{e}^{u_{1}\left(\eta_{1}\right)}-\bar{D} \omega+\phi_{3}}{C\left(\bar{D} \omega-\phi_{3}\right)} .
$$

By (3.3) and the assumption in Theorem 3, we have 


$$
\mathrm{e}^{u_{3}\left(\xi_{3}\right)} \leq \frac{\left[\bar{E} \omega-B\left(\bar{D} \omega-\phi_{3}\right)\right] \mathrm{e}^{w_{12}}-\bar{D} \omega+\phi_{3}}{C\left(\bar{D} \omega-\phi_{3}\right)} .
$$

We have

$$
u_{3}\left(\xi_{3}\right) \leq \ln \frac{\left[\bar{E} \omega-B\left(\bar{D} \omega-\phi_{3}\right)\right] \mathrm{e}^{W_{12}}-\bar{D} \omega+\phi_{3}}{C\left(\bar{D} \omega-\phi_{3}\right)} \triangleq G_{32} .
$$

By (2.9) and (3.11), we have

$$
u_{3}(t) \leq u_{3}\left(\xi_{3}\right)-\int_{0}^{\omega}\left|\dot{u}_{3}(t)\right| \mathrm{d} t-\left|\phi_{3}\right| \leq G_{32}-2 \bar{D} \omega+\phi_{3}-\left|\phi_{3}\right| \triangleq W_{32} .
$$

Therefore, by (3.9) and (3.12), we have

$$
\max _{t \in[0, \omega]}\left|u_{3}(t)\right| \leq \max \left\{\left|W_{31}\right|,\left|W_{32}\right|\right\} .
$$

This completes the proof of Lemma 5 .

Proof of Lemma 6. By (2.3) and (2.10), we have

$$
\bar{r} \omega+\phi_{1} \leq \frac{\bar{r} \omega \mathrm{e}^{u_{1}\left(\eta_{1}\right)}}{k}+\frac{\bar{a} \omega \mathrm{e}^{u_{2}\left(\eta_{2}\right)}}{1+b \mathrm{e}^{u_{1}\left(\xi_{1}\right)}}+\frac{\bar{A} \omega \mathrm{e}^{u_{3}\left(\eta_{3}\right)}}{1+B \mathrm{e}^{u_{1}\left(\xi_{1}\right)}+C \mathrm{e}^{u_{3}\left(\eta_{3}\right)}} .
$$

By multiplying both sides of (3.13) by $\exp \left\{u_{1}\left(\xi_{1}\right)\right\}$, it follows that

$$
\left(\bar{r} \omega+\phi_{1}\right) \mathrm{e}^{u_{1}\left(\xi_{1}\right)} \leq \frac{\bar{r} \omega \mathrm{e}^{u_{1}\left(\xi_{1}\right)} \mathrm{e}^{u_{1}\left(\eta_{1}\right)}}{k}+\frac{\bar{a} \omega \mathrm{e}^{u_{1}\left(\xi_{1}\right)} \mathrm{e}^{u_{2}\left(\eta_{2}\right)}}{1+b \mathrm{e}^{u_{1}\left(\xi_{1}\right)}}+\frac{\bar{A} \omega \mathrm{e}^{u_{1}\left(\xi_{1}\right)} \mathrm{e}^{u_{3}\left(\eta_{3}\right)}}{1+B \mathrm{e}^{u_{1}\left(\xi_{1}\right)}+C \mathrm{e}^{u_{3}\left(\eta_{3}\right)}} .
$$

By (3.1) and (3.7), we have

$$
\left(\bar{r} \omega+\phi_{1}\right) \mathrm{e}^{u_{1}\left(\xi_{1}\right)} \leq \frac{\bar{r} \omega \mathrm{e}^{u_{1}\left(\xi_{1}\right)} \mathrm{e}^{u_{1}\left(\eta_{1}\right)}}{k}+\frac{\bar{a} \omega\left(\bar{d} \omega-\phi_{2}\right) \mathrm{e}^{u_{2}\left(\eta_{2}\right)}}{\bar{e} \omega}+\frac{\bar{A} \omega\left(\bar{D} \omega-\phi_{3}\right) \mathrm{e}^{u_{3}\left(\eta_{3}\right)}}{\bar{E} \omega} .
$$

It follows that

$$
\frac{\bar{a} \omega\left(\bar{d} \omega-\phi_{2}\right)}{\bar{e} \omega} \mathrm{e}^{u_{2}\left(\eta_{2}\right)} \geq \mathrm{e}^{u_{1}\left(\xi_{1}\right)}\left[\left(\bar{r} \omega+\phi_{1}\right)-\frac{\bar{r} \omega \mathrm{e}^{u_{1}\left(\eta_{1}\right)}}{k}\right]-\frac{\bar{A} \omega\left(\bar{D} \omega-\phi_{3}\right) \mathrm{e}^{u_{3}\left(\eta_{3}\right)}}{\bar{E} \omega} .
$$

By (3.3), (3.6), (3.12), we have

$$
\frac{\bar{a} \omega\left(\bar{d} \omega-\phi_{2}\right)}{\bar{e} \omega} \mathrm{e}^{u_{2}\left(\eta_{2}\right)} \geq \mathrm{e}^{W_{11}}\left[\left(\bar{r} \omega+\phi_{1}\right)-\frac{\bar{r} \omega \mathrm{e}^{W_{12}}}{k}\right]-\frac{\bar{A} \omega\left(\bar{D} \omega-\phi_{3}\right) \mathrm{e}^{W_{32}}}{\bar{E} \omega} .
$$

It follows that

$$
\begin{aligned}
& u_{2}\left(\eta_{2}\right) \\
& \geq \ln \left\{\frac{\bar{e} \omega}{\bar{a} \omega\left(\bar{d} \omega-\phi_{2}\right)}\left[\mathrm{e}^{W_{11}}\left(\left(\bar{r} \omega+\phi_{1}\right)-\frac{\bar{r} \omega \mathrm{e}^{W_{12}}}{k}\right)-\frac{\bar{A} \omega\left(\bar{D} \omega-\phi_{3}\right) \mathrm{e}^{W_{32}}}{\bar{E} \omega}\right]\right\} \\
& \triangleq G_{21} .
\end{aligned}
$$

Therefore, by (3.14), we have

$$
u_{2}(t) \geq u_{2}\left(\eta_{2}\right)-\int_{0}^{\omega}\left|\dot{u}_{2}(t)\right| \mathrm{d} t-\left|\phi_{2}\right| \geq G_{21}-2 \bar{d} \omega+\phi_{2}-\left|\phi_{2}\right| \triangleq W_{21} .
$$

On the other hand, we have

$$
\bar{r} \omega+\phi_{1} \geq \frac{\bar{r} \omega \mathrm{e}^{u_{1}\left(\xi_{1}\right)}}{k}+\frac{\bar{a} \omega \mathrm{e}^{u_{2}\left(\xi_{2}\right)}}{1+b^{u_{1}\left(\eta_{1}\right)}}+\frac{\bar{A} \omega \mathrm{e}^{u_{3}\left(\xi_{3}\right)}}{1+B \mathrm{e}^{u_{1}\left(\eta_{1}\right)}+C \mathrm{e}^{u_{3}\left(\xi_{3}\right)}} .
$$


By multiplying both sides of (3.16) by $\exp \left\{u_{1}\left(\eta_{1}\right)\right\}$, it follows that

$$
\left(\bar{r} \omega+\phi_{1}\right) \mathrm{e}^{u_{1}\left(\eta_{1}\right)} \geq \frac{\bar{r} \omega \mathrm{e}^{u_{1}\left(\eta_{1}\right)} \mathrm{e}^{u_{1}\left(\xi_{1}\right)}}{k}+\frac{\bar{a} \omega \mathrm{e}^{u_{1}\left(\eta_{1}\right)} \mathrm{e}^{u_{2}\left(\xi_{2}\right)}}{1+b^{u_{1}\left(\eta_{1}\right)}}+\frac{\bar{A} \omega \mathrm{e}^{u_{1}\left(\eta_{1}\right)} \mathrm{e}^{u_{3}\left(\xi_{3}\right)}}{1+B \mathrm{e}^{u_{1}\left(\eta_{1}\right)}+C \mathrm{e}^{u_{3}\left(\xi_{3}\right)}} .
$$

By (3.4) and (3.10), we have

$$
\left(\bar{r} \omega+\phi_{1}\right) \mathrm{e}^{u_{1}\left(\eta_{1}\right)} \geq \frac{\bar{r} \omega \mathrm{e}^{u_{1}\left(\eta_{1}\right)} \mathrm{e}^{u_{1}\left(\xi_{1}\right)}}{k}+\frac{\bar{a} \omega\left(\bar{d} \omega-\phi_{2}\right) \mathrm{e}^{u_{2}\left(\xi_{2}\right)}}{\bar{e} \omega}+\frac{\bar{A} \omega\left(\bar{D} \omega-\phi_{3}\right) \mathrm{e}^{u_{3}\left(\xi_{3}\right)}}{\bar{E} \omega} .
$$

It follows that

$$
\frac{\bar{a} \omega\left(\bar{d} \omega-\phi_{2}\right)}{\bar{e} \omega} \mathrm{e}^{u_{2}\left(\xi_{2}\right)} \leq \mathrm{e}^{u_{1}\left(\eta_{1}\right)}\left[\left(\bar{r} \omega+\phi_{1}\right)-\frac{\bar{r} \omega \mathrm{e}^{u_{1}\left(\xi_{1}\right)}}{k}\right]-\frac{\bar{A} \omega\left(\bar{D} \omega-\phi_{3}\right) \mathrm{e}^{u_{3}\left(\xi_{3}\right)}}{\bar{E} \omega} .
$$

By (3.3), (3.6), (3.9), we have

$$
\frac{\bar{a} \omega\left(\bar{d} \omega-\phi_{2}\right)}{\bar{e} \omega} \mathrm{e}^{u_{2}\left(\xi_{2}\right)} \leq \mathrm{e}^{W_{12}}\left[\left(\bar{r} \omega+\phi_{1}\right)-\frac{\bar{r} \omega \mathrm{e}^{W_{11}}}{k}\right]-\frac{\bar{A} \omega\left(\bar{D} \omega-\phi_{3}\right) \mathrm{e}^{W_{31}}}{\bar{E} \omega} .
$$

It follows that

$$
\begin{aligned}
& u_{2}\left(\xi_{2}\right) \\
& \leq \ln \left\{\frac{\bar{e} \omega}{\bar{a} \omega\left(\bar{d} \omega-\phi_{2}\right)}\left[\mathrm{e}^{W_{12}}\left(\left(\bar{r} \omega+\phi_{1}\right)-\frac{\bar{r} \omega \mathrm{e}^{W_{11}}}{k}\right)-\frac{\bar{A} \omega\left(\bar{D} \omega-\phi_{3}\right) \mathrm{e}^{W_{31}}}{\bar{E} \omega}\right]\right\} \\
& \triangleq G_{22} .
\end{aligned}
$$

Therefore, by (3.17), we have

$$
u_{2}(t) \leq u_{2}\left(\xi_{2}\right)+\int_{0}^{\omega}\left|\dot{u}_{2}(t)\right| \mathrm{d} t+\left|\phi_{2}\right| \leq G_{22}+2 \bar{d} \omega-\phi_{2}+\left|\phi_{2}\right| \triangleq W_{22} .
$$

Therefore, by (3.15) and (3.18), we have

$$
\max _{t \in[0, \omega]}\left|u_{3}(t)\right| \leq \max \left\{\left|W_{21}\right|,\left|W_{22}\right|\right\} .
$$

This completes the proof of Lemma 6.

\section{Theorems and Proof}

Our main result of this paper is as follows:

Theorem 6. If (H1)-(H6) hold, then system (1.5) has at least one $\omega$-periodic positive solution.

Proof. Based on the Lemmas 4-6, it can be seen that the constants $W_{i}(i=1,2,3)$, are independent of $\lambda$. Moreover, it can be verified that the following system of algebraic equations

$$
\left\{\begin{array}{l}
\bar{r}\left[1-\frac{x}{k}\right]-\frac{\bar{a} y}{1+b x}-\frac{\bar{A} z}{1+B x+C z}=-\frac{\phi_{1}}{\omega}, \\
-\bar{d}+\frac{\bar{e} x}{1+b x}=-\frac{\phi_{2}}{\omega}, \\
-\bar{D}+\frac{\bar{E} x}{1+B x+C z}=-\frac{\phi_{3}}{\omega},
\end{array}\right.
$$


has a unique positive solution $\left[x^{*}(t), y^{*}(t), z^{*}(t)\right]^{\mathrm{T}}$.

Denote $W=W_{1}+W_{2}+W_{3}+W_{4}$, where $W_{4}>0$ is taken to be sufficiently large such that

$$
\left\|\left(\ln x^{*}, \ln y^{*}, \ln z^{*}\right)\right\|_{X}=\sup _{t \in[0, \omega]}\left|\ln x^{*}\right|+\sup _{t \in[0, \omega]}\left|\ln y^{*}\right|+\sup _{t \in[0, \omega]}\left|\ln z^{*}\right|<W_{4} .
$$

Define $\Omega=\left\{u=\left(u_{1}, u_{2}, u_{3}\right)^{\mathrm{T}}: u_{1}, u_{2}, u_{3} \in P C_{\omega}^{1},\|u\|_{X}<W\right\}$. It is clear that $\Omega$ verifies the requirement (a) of the Lemma 2. When $u \in \partial \Omega \cap \operatorname{Ker} L=\partial \Omega \cap \mathbb{R}^{3}$, $u$ is a constant vector in $\mathbb{R}^{3}$ with $\|u\|_{X}=W$. Then for $u \in \partial \Omega \cap \operatorname{Ker} L$,

$$
Q N u=\left(\begin{array}{c}
\bar{r}\left[1-\frac{\mathrm{e}^{u_{1}}}{k}\right]-\frac{\bar{a} \mathrm{e}^{u_{2}}}{1+b \mathrm{e}^{u_{1}(t)}}-\frac{\bar{A} \mathrm{e}^{u_{3}}}{1+B \mathrm{e}^{u_{1}(t)}+C \mathrm{e}^{u_{3}}}+\frac{\phi_{1}}{\omega} \\
-\bar{d}+\frac{\bar{e} \mathrm{e}^{u_{1}(t)}}{1+b \mathrm{e}^{u_{1}(t)}}+\frac{\phi_{2}}{\omega} \\
-\bar{D}+\frac{\bar{E} \mathrm{e}^{u_{1}(t)}}{1+B \mathrm{e}^{u_{1}(t)}+C \mathrm{e}^{u_{3}}}+\frac{\phi_{3}}{\omega}
\end{array}\right) \neq 0 .
$$

The isomorphism $J$ of $\operatorname{Im} Q$ onto $\operatorname{Ker} L$ may be defined by

$$
J: \operatorname{Im} Q \rightarrow X,\left[\left(\begin{array}{l}
\varphi_{1} \\
\varphi_{2} \\
\varphi_{3}
\end{array}\right),\left(\begin{array}{l}
0 \\
0 \\
0
\end{array}\right), \cdots,\left(\begin{array}{l}
0 \\
0 \\
0
\end{array}\right)\right] \rightarrow\left(\begin{array}{l}
\varphi_{1} \\
\varphi_{2} \\
\varphi_{3}
\end{array}\right),
$$

then

$$
J Q N u=\left(\begin{array}{c}
\bar{r}\left[1-\frac{\mathrm{e}^{u_{1}}}{k}\right]-\frac{\bar{a} \mathrm{e}^{u_{2}}}{1+b \mathrm{e}^{u_{1}(t)}}-\frac{\bar{A} \mathrm{e}^{u_{3}}}{1+B \mathrm{e}^{u_{1}(t)}+C \mathrm{e}^{u_{3}}}+\frac{\phi_{1}}{\omega} \\
-\bar{d}+\frac{\bar{e} \mathrm{e}^{u_{1}(t)}}{1+b \mathrm{e}^{u_{1}(t)}}+\frac{\phi_{2}}{\omega} \\
-\bar{D}+\frac{\bar{E} \mathrm{e}^{u_{1}(t)}}{1+B \mathrm{e}^{u_{1}(t)}+C \mathrm{e}^{u_{3}}}+\frac{\phi_{3}}{\omega}
\end{array}\right)
$$

On the other hand, by the definition of topology degree, direct calculation yields

$$
\operatorname{deg}\{J Q N u, \Omega \cap \operatorname{Ker} L, 0\}=\operatorname{sign}\left|\begin{array}{lll}
f_{u_{1}} & f_{u_{2}} & f_{u_{3}} \\
g_{u_{1}} & 0 & 0 \\
h_{u_{1}} & 0 & h_{u_{3}}
\end{array}\right| \text {, }
$$

where $f_{u_{1}}=\frac{\bar{r} \mathrm{e}^{u_{1}}}{k}+\frac{\bar{a} b \mathrm{e}^{u_{1}+u_{2}}}{\left[1+b \mathrm{e}^{u_{1}}\right]^{2}}+\frac{\bar{A} B \mathrm{e}^{u_{1}+u_{3}}}{\left[1+B \mathrm{e}^{u_{1}(t)}+C \mathrm{e}^{u_{3}}\right]^{2}}, \quad f_{u_{2}}=-\frac{\bar{a} \mathrm{e}^{u_{2}}}{1+b \mathrm{e}^{u_{1}}}<0$,

$$
\begin{aligned}
& f_{u_{3}}=\frac{\bar{A} \mathrm{e}^{u_{3}}\left[1+B \mathrm{e}^{u_{1}}\right]}{\left[1+B \mathrm{e}^{u_{1}}+C \mathrm{e}^{u_{3}}\right]^{2}}, \quad g_{u_{1}}=\frac{\bar{e} \mathrm{e}^{u_{1}}}{\left[1+b \mathrm{e}^{u_{1}}\right]^{2}}>0, h_{u_{1}}=\frac{\bar{E} \mathrm{e}^{u_{1}}\left[1+C \mathrm{e}^{u_{3}}\right]}{\left[1+B \mathrm{e}^{u_{1}(t)}+C \mathrm{e}^{u_{3}}\right]^{2}}, \\
& h_{u_{3}}=-\frac{C \bar{E} \mathrm{e}^{u_{1}+u_{3}}}{\left[1+B \mathrm{e}^{u_{1}}+C \mathrm{e}^{u_{3}}\right]^{2}}<0 .
\end{aligned}
$$

Therefore, we have 


$$
\operatorname{deg}\{J Q N u, \Omega \cap \operatorname{Ker} L, 0\}=\operatorname{sign}\left|\begin{array}{lll}
f_{u_{1}} & f_{u_{2}} & f_{u_{3}} \\
g_{u_{1}} & 0 & 0 \\
h_{u_{1}} & 0 & h_{u_{3}}
\end{array}\right|=-1 \neq 0 .
$$

So far we have proved that $\Omega$ satisfies all the requirements in Lemma 2 . Therefore, system (2.1) has at least one $\omega$-periodic solution. Hence, by the transformation $x(t)=\mathrm{e}^{u_{1}(t)}, y(t)=\mathrm{e}^{u_{2}(t)}, z(t)=\mathrm{e}^{u_{3}(t)}$, system (1.5) has at least one positive $\omega$-periodic solution, this completes the proof.

Remark 7. In [7], the conditions of the existence of positive periodic solutions were obtained for nonautonomous delayed predator-prey system such as system (1.4). However, it did not consider the impulsive impacts. This paper discusses the nonautonomous delayed predator-prey system with impulsive effects and obtains the sufficient conditions of the existence of positive periodic solutions of system (1.5) by employing the method of the continuation theorem of Gaines and Mawhin. Therefore, the work of this paper extends the main results in literature [7].

\section{Conclusions and Future Works}

It is usually observed that population densities in the real world tend to fluctuate. Therefore, modeling population interactions and understanding this oscillatory phenomenon are a very basic and important ecological problem. Although much progress has been made in the study of modelling and understanding three species predator-prey systems, models such as (1.1)-(1.3) have been largely discussed by assuming that the environment is constant, which is indeed rarely the case in real life. Naturally, more realistic and interesting models with three species interactions should take into account the seasonality of the changing environment, the effects of time delays and artificially regularly put predators and prey. Therefore, it is interesting and important to study systems with impulses and periodic delays (1.5). In this paper, based on the powerful and effective coincidence degree theory, the existence of positive periodic solutions for predator-prey systems with impulses and periodic delays (1.5) is obtained under suitable conditions. In particular, in the system (1.5), when there is no impulses, that is $\phi_{i k} \equiv 0(i=1,2,3)$, the results are obtained in [4].

Of course, there are some improvements in this article to explore further. For instance,

1) Positive almost positive periodic solutions of nonautonomous delayed predator-prey system with pulse controls are meaningful to discuss.

2) Stability of nonautonomous delayed predator-prey system with pulse controls should be studied in the future.

3) Other dynamical behaviors of nonautonomous delayed predator-prey system with pulse controls should be further investigated.

\section{Acknowledgements}

This work is supported by Science and Technology Plan Project of Guizhou 
Province (Contract No.: Qiankehe LH [2017] No. 7031).

\section{Conflicts of Interest}

The authors declare no conflicts of interest regarding the publication of this paper.

\section{References}

[1] Rihan, F.A., Lakshmanan, S., Hashish, A.H., Rakkiyappan, R. and Ahmed, E. (2015) Fractional-Order Delayed Predator-Prey Systems with Holling Type-II Functional Response. Nonlinear Dynamics, 80, 777-789. https://doi.org/10.1007/s11071-015-1905-8

[2] El-Saka, H.A.A., Lee, S. and Jang, B. (2019) Dynamic Analysis of Fractional-Order Predator-Prey Biological Economic System with Holling Type II Functional Response. Nonlinear Dynamics, 96, 407-416. https://doi.org/10.1007/s11071-019-04796-y

[3] Du, Y., Niu, B. and Wei, J. (2019) Two Delays Induce Hopf Bifurcation and Double Hopf Bifurcation in a Diffusive Leslie-Gower Predator-Prey System. Chaos, 29, Article ID: 013101. https://doi.org/10.1063/1.5078814

[4] Lotka, A. (1925) Elements of Physical Biology. Williams and Wilkins, Baltimore.

[5] Volterra, V. (1926) Variazioni e fluttuaziono del numero di individui in specie animali conviventi. Memoria della Reale Accademia Nazionale dei Lincei, 2, 31-113.

[6] Liu, W., Xiao, D. and Yi, Y. (2003) Relaxation Oscillations in a Class of Predator Prey Systems. Journal of Differential Equations, 188, 306-331. https://doi.org/10.1016/S0022-0396(02)00076-1

[7] Lin, G. and Hong, Y. (2009) Periodic Solutions in Non Autonomous Predator Prey System with Delays. Nonlinear Analysis: Real World Applications, 10, 1589-1600. https://doi.org/10.1016/j.nonrwa.2008.02.003

[8] Kuang, Y. (1993) Delay Differential Equations with Applications in Population Dynamics. Academic Press, Boston.

[9] Li, Y. and Kuang, Y. (2001) Periodic Solutions of Periodic Delay Lotka-Volterra Equations and Systems. Journal of Mathematical Analysis and Applications, 255, 260-280. https://doi.org/10.1006/jmaa.2000.7248

[10] Zhao, T., Kuang, Y. and Smith, H.L. (1997) Global Existence of Periodic Solutions in a Class of Delayed Gause-Type Predator-Prey Systems. Nonlinear Analysis. Real World Applications, 28, 1373-1394. https://doi.org/10.1016/0362-546X(95)00230-S

[11] Kar, T. and Pahari, U. (2007) Modelling and analysis of a prey-predator system with stage-structure and harvesting, Nonlinear Analysis: Real World Applications, 8, 601-609. https://doi.org/10.1016/j.nonrwa.2006.01.004

[12] Song, Y. and Yuan, S. (2006) Bifurcation Analysis in a Predator-Prey System with Time Delay. Nonlinear Analysis: Real World Applications, 7, 265-284. https://doi.org/10.1016/j.nonrwa.2005.03.002

[13] Xiao, D. and Ruan, S. (2001) Multiple Bifurcations in a Delayed Predator-Prey System with Nonmonotonic Functional Response. Journal of Differential Equations, 176, 494-510. https://doi.org/10.1006/jdeq.2000.3982

[14] Jones, C. (1995) Geometric Singular Perturbation Theory. In: Dynamical Systems, Lecture Notes in Math, Vol. 1609, Springer-Verlag, Berlin, 44-120. https://doi.org/10.1007/BFb0095239

[15] Lin, G. and Yuan, R. (2005) Periodic Solution for a Predator-Prey System with Dis- 
tributed Delay. Mathematical and Computer Modelling, 42, 959-966.

https://doi.org/10.1016/j.mcm.2005.05.015

[16] Lin, G. and Yuan, R. (2006) Periodic Solutions for Equations with Distributed Delays. Proceedings of the Royal Society of Edinburgh Section A, 136, 1317-1325. https://doi.org/10.1017/S030821050000500X

[17] Bainov, D. and Simeonov, P. (1993) Impulsive Differential Equations: Periodic Solutions and Applications. Monographs and Surveys in Pure and Applied Mathematics, Vol. 66, Routledge, London.

[18] Gaines, R. and Mawhin, J. (1977) Coincidence Degree and Nonlinear Differential Equations. Springer, Berlin. https://doi.org/10.1007/BFb0089537 